\title{
Dispersive charge carrier mobility in a surface oxide layer
}

\author{
Gabor Nagy and Robert Schiller \\ Central Research Institute for Physics, Atomic Energy Research Institute, P.O.B. 49, \\ Budapest H-1525, Hungary
}

Received 30th July 2001, Accepted 4th December 2001

First published as an Advance Article on the web 29th January 2002

\begin{abstract}
The electrode impedance spectrum of the oxide produced on a $\mathrm{Zr}-1 \% \mathrm{Nb}$ surface in an aqueous solution at $290^{\circ} \mathrm{C}$ is analysed as a function of temperature between $20^{\circ} \mathrm{C}$ and $290^{\circ} \mathrm{C}$. The portion of the spectrum, relevant to the oxide, is treated in terms of a parallel CPE $\| R_{\text {ox }}$ equivalent circuit (CPE denoting constant phase element, $R_{\text {ox }}$ ohmic resistor). Considering also the effect of temperature CPE is understood as a dispersive resistance described by the continuous time random walk theory of Scher and Lax. Distinction is made between the kinetics of oxide growth and of non-Faraday charge transport, the time constant of the latter being obtained directly from impedance data.
\end{abstract}

\section{Introduction}

This work was prompted by our kinetic experiments on thermal oxide growth on the surface of the corrosion resistant alloy $\mathrm{Zr}-1 \% \mathrm{Nb}$ in high temperature aqueous solutions. Electrode kinetics, as studied by impedance analysis, is usually described in terms of equivalent electric circuits composed of passive linear elements. ${ }^{1}$ Linearity in this context means that the impedances of the elements are independent of the perturbing voltage, whereas the kinetics, i.e. the time dependence of the potential versus current functions, is characterized by the frequency dependences of the impedances, $Z(\omega)$. The elements are usually denoted by the names used in the theory of electric circuits: a frequency independent element is called ohmic resistance, $R$; that with a $Z_{C} \propto 1 /(\mathrm{i} \omega)$ dependence is called capacitance; a third one, occurring relatively rarely, where $Z_{L} \propto \mathrm{i} \omega$ prevails is called inductance. One can usually attribute elementary transport processes to each of these elements and the assignments, at least in simple cases, are easy to visualize.

More complicated processes involve less pictorial descriptions. For example the frequency dependence of a diffusion controlled process is given as a Warburg impedance, $W(\omega)=A(\mathrm{i} \omega)^{-1 / 2}$, the passive electric analogy of which can only be given by making use of an infinitely large electric circuit.

A family of impedances can be described by the so-called constant phase element, $\mathrm{CPE}$, which is given as

$$
Z_{\mathrm{CPE}}=(1 / \sigma)(\mathrm{i} \omega)^{-\alpha},
$$

where $0 \leqslant \alpha \leqslant 1$ holds. Usually $Z_{\mathrm{CPE}}$ appears in the equivalent circuits parallel with an ohmic impedance, $R$. The result is

$$
1 / Z_{\mathrm{CPER}}=(1 / R)+\sigma(\mathrm{i} \omega)^{\alpha}=(1 / R)\left[1+(\mathrm{i} \omega \tau)^{\alpha}\right] .
$$

where the notation $\tau=(R \sigma)^{1 / \alpha}$ is used.

This element, ubiquitous as it is in the description of complicated electrode processes, is difficult to interpret in terms of a single model of general validity. Given the versatility of $Z_{\mathrm{CPE}}$ in view of its two free parameters, $\sigma$ and $\alpha$, one might be tempted to consider it as an easy-to-handle analytical approximation to a number of physically more or less distinct processes. $Z_{\mathrm{CPE}}$ is usually regarded as a dispersive capacitance the dispersion of which can be attributed to dielectric relaxation or to geometrical factors. Obviously, no interpretation can rest solely on the frequency spectra, one has also to analyse the effect of other experimental parameters.

The aim of this paper is to offer a possible rationalization of our findings $\mathrm{s}^{2}$ on the oxidation of the alloy $\mathrm{Zr}-1 \% \mathrm{Nb}$ in the kinetics of which $Z_{\text {CPER }}$ plays an important role. The experimental parameter, beyond frequency and electrode potential, is temperature. The $Z_{\mathrm{CPER}}$ element will be interpreted as a dispersive resistance by making use of the theory of continuous time random walk (CTRW).

\section{Experimental}

The oxidation of the $\mathrm{Zr}$ alloy containing $1 \% \mathrm{Nb}$, a metal used as fuel element cladding in Russian-made pressure water reactors type VVER, was investigated. Tubular samples connected to electrical feedthroughs were placed into a PARR 4532 autoclave which was filled with an aqueous solution containing 0.8 $\mathrm{g} \mathrm{kg}^{-1}$ boric acid, $5 \mathrm{mg} \mathrm{kg}^{-1}$ ammonium hydroxide and $5 \mathrm{mg}$ $\mathrm{kg}^{-1}$ potassium hydroxide, a mixture, typical of a VVER primary circuit. After de-aeration by argon gas bubbling the samples were kept in the solution at $290^{\circ} \mathrm{C}$ for up to 140 days. The progress of oxidation was monitored by in situ electrochemical impedance spectroscopy (EIS). Periodically, the system was cooled to room temperature in order to determine the temperature dependence of the spectrum.

Solartron 1286 or Electroflex EF453 potentiostats coupled to a Solartron 1250 frequency response analyser were used for EIS measurements in the frequency range $64 \mathrm{kHz}-0.1 \mathrm{~Hz}$ (sometimes $0.001 \mathrm{~Hz}$ ) with an ac amplitude of $5 \mathrm{mV}$. Our previous ex situ results ${ }^{2}$ proved $\mathrm{Zr}-1 \% \mathrm{Nb}$ to be electrochemically inert under the present experimental conditions. Moreover, there exists a broad potential range around zero (vs. NHE) where the measured impedance spectra are independent of electrode potential, a fact which enabled us to perform in situ EIS measurements in a two-electrode arrangement with fixed zero potential between the two electrodes. The EIS were taken daily at $290^{\circ} \mathrm{C}$, and also during each heating-up period at $20^{\circ} \mathrm{C}, 110^{\circ} \mathrm{C}, 200^{\circ} \mathrm{C}$ and $290^{\circ} \mathrm{C}$. Further experimental details are given in ref. 2 .

The measurements clearly show that the impedance spectra reveal the development of the oxide layer on $\mathrm{Zr}-1 \% \mathrm{Nb}$. While the non-oxidised (metallic) sample has a simple spectrum indicating a single interfacial process, very soon after the start of 


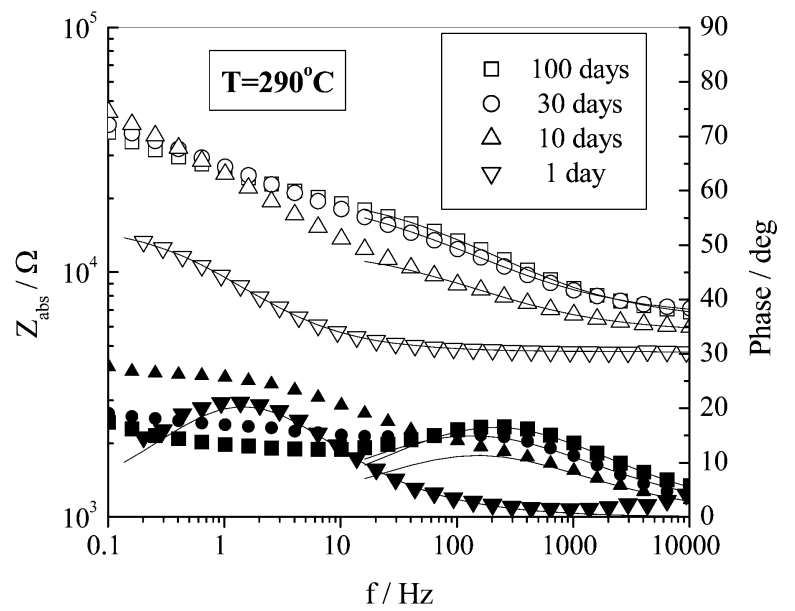

Fig. 1 Variation of in situ impedance spectra of $\mathrm{Zr}-1 \% \mathrm{Nb}$ at $290^{\circ} \mathrm{C}$ with the duration of oxidation (open symbols: magnitude; filled symbols: phase angle). The solid lines are the results of non-linear fits for the intervals given by the end points of the lines.

oxidation the shape of the spectra changes as shown in Fig. 1. On the phase angle curve a new peak appears at higher frequencies whereas the low frequency peak, characteristic of the non-oxidised sample, gradually disappears. As oxidation proceeds the high frequency peak becomes more pronounced. The spectra are temperature dependent (Fig. 2); the high frequency part changes particularly strongly.

We analysed our results by fitting a number of different equivalent circuits only to find that the spectra are not informative enough to enable one to select a unique description of the results. However, a Randles-circuit, $\left(-R_{\mathrm{s}}-\left[C \|\left(R_{\mathrm{p}}-W\right)\right]-\right)$, in series with an $R \| \mathrm{CPE}$ type element seemed to be the best choice considering also the physical picture involved: the Randles-circuit describes a diffusion-controlled interfacial process whereas the $R \| \mathrm{CPE}$ element is characteristic of a large family of dielectric materials (with the inevitable resistance for electron transport across the layer). Unfortunately, the fitted parameters turned out to be strongly correlated; hence we cannot consider this circuit to be unique and representative.

Therefore, instead of trying to discuss the entire spectrum we focus our attention on the high frequency region. In view of what was said above it can be reasonably assigned to processes connected with the oxide layer. We analyse the $10 \mathrm{~Hz}-$

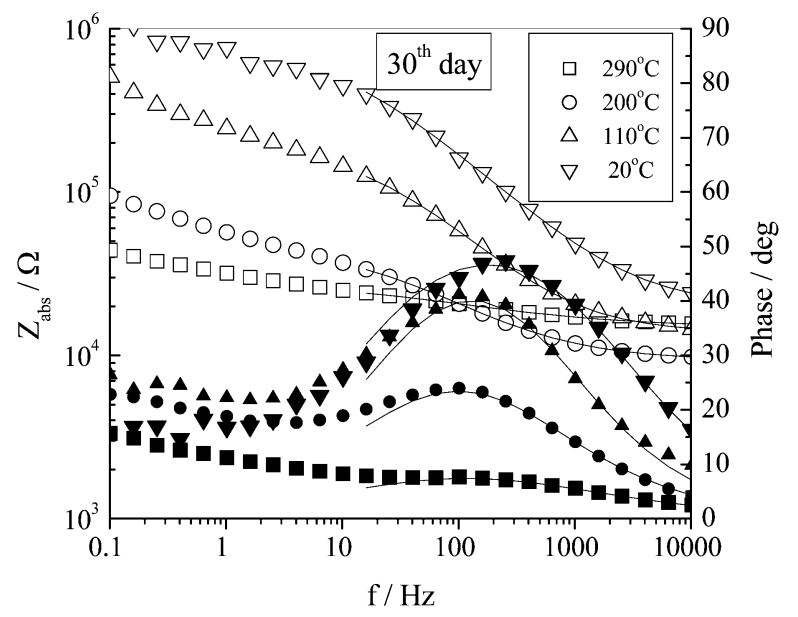

Fig. 2 In situ impedance spectra of $\mathrm{Zr}-1 \% \mathrm{Nb}$, oxidised for 30 days, taken at different temperatures (open symbols: magnitude; filled symbols: phase angle). The solid lines are the results of non-linear fits for the intervals given by the end points of the lines.

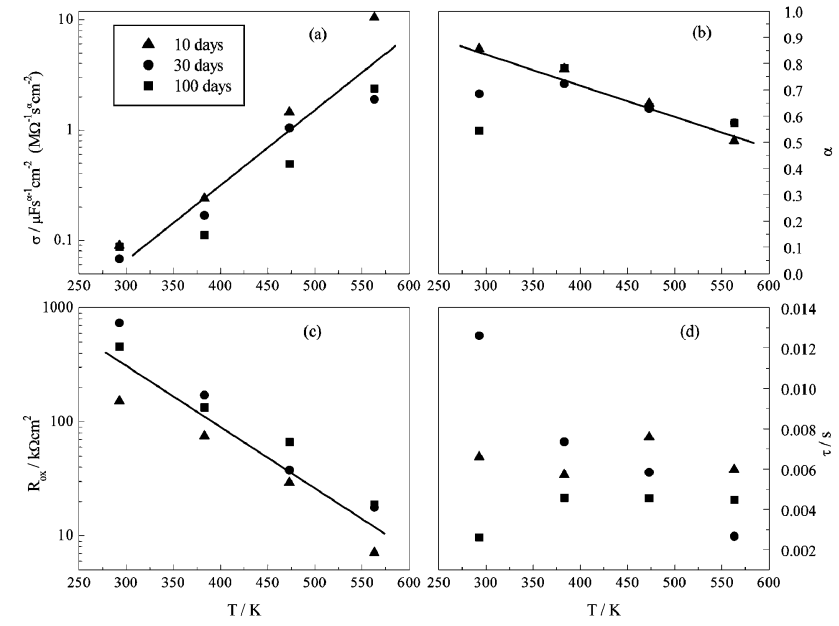

Fig. 3 Temperature dependence of the parameters obtained by fitting the impedance spectra of oxidised $\mathrm{Zr}-1 \% \mathrm{Nb}$ with the $R_{\mathrm{s}}-\mathrm{CPE} \| R_{\mathrm{ox}}$ equivalent circuit in the $10 \mathrm{~Hz}-10 \mathrm{kHz}$ frequency range. (a) CPE coefficient, $\sigma$; (b) CPE exponent, $\alpha$; (c) $R_{\text {ox }}$; (d) characteristic time, $\tau$ (see text). Filled triangles: sample oxidised for 10 days; filled circles: sample oxidised for 50 days; filled squares: sample oxidised for 100 days. The solid lines are to guide the eye.

$10 \mathrm{kHz}$ frequency range in terms of a $Z_{\mathrm{CPER}}$ element by fitting eqn. (2), whereas for the non-oxidised sample the $0.1 \mathrm{~Hz}-10$ $\mathrm{kHz}$ range was chosen.

The parameters as a function of temperature are given in Fig. 3. The three points of any given temperature correspond to three different durations of oxidation. Here one sees that $\sigma$ increases (Fig. 3(a)) and the parallel ohmic resistance, $R_{\mathrm{ox}}$, decreases markedly (Fig. 3(c)) with increasing temperature, whereas $\alpha$ decreases slightly with temperature and $\tau=\left(R_{\mathrm{ox}} \sigma\right)^{1 / \alpha}$ is almost independent of temperature (Figs. 3 (b) and (d)). These trends hold invariably for samples which were oxidized for 10 to 100 days.

\section{Discussion}

Usually $Z_{\mathrm{CPE}}(\omega)$ is considered to be a dispersive capacitance. In this picture $\sigma$ is regarded as a pseudo-capacity, although its dimension differs from the farad, and $\alpha$ is the measure of the capacitive nature of the element: if $\alpha=1$ the element is an ideal capacitor, if $\alpha=0$ it behaves as a frequency independent ohmic resistor, whereas if $\alpha=-1$ it behaves as an inductance. (Note that there are known cases when $Z_{\mathrm{CPE}}$ cannot be treated this way; e.g. the Warburg impedance, which is connected with diffusion, is a CPE element with $\alpha=0.5$ ).

The temperature dependences of the fitted parameters, observed in the present experiments, contravene any explanation, which would rest on a capacitor model. The capacity of a condenser of any geometry is proportional to the relative permittivity of the dielectric material, $\varepsilon$. Since $\varepsilon$ usually decreases with increasing temperature, (with phase transitions representing obvious but rare exceptions), increasing temperature would lead to a decrease in capacity i.e. within the framework of the dispersive capacity model $\sigma$ is expected to decrease as $T$ increases. Our observations contradict this expectation.

Hence it seems reasonable to make use of a model of dispersive resistance instead of dispersive capacitance. As Macdonald $^{3}$ pointed out the theory of continuous time random walk (CTRW) by Scher and $\mathrm{Lax}^{4}$ can be applied to the present problem of charge transport in solid disordered media. Comp$\mathrm{te}^{5 \mathrm{a}}$ and Bisquert and Compte ${ }^{5 \mathrm{~b}}$ analysed a number of models and theories of anomalous diffusion in terms of fractional calculus, with a view to electrochemical impedance spectra. They proved that the mechanism and conditions of diffusion define 
the spectrum, predicting CPE behaviour under certain circumstances. Let us first quote the basic relationships between resistance, $R$, specific resistivity, $\rho$, charge density, $n$, mobility, $\mu$, and diffusion coefficient, $D$, as

$$
R^{-1}=g \rho^{-1}=g n \mu=\frac{e}{k T} g n D
$$

where $g$ denotes the geometrical factor of the sample, and $e, k$, $T$ are elementary charge, Boltzmann constant and absolute temperature, respectively. According to Scher and Lax if the random walk of the charge carriers is characterised by some distribution of the waiting times between successive jumps, then $D$ is a function of time or of angular frequency, $\omega$. Let $\psi(t) d t$ denote the normalised probability of a jump to take place between $t$ and $t+d t$; here $\psi(t)$ is called the waiting time distribution function with $\tilde{\psi}(\mathrm{i} \omega)$ denoting its Fourier transform. With this notation the dispersion of $D$ can be written as ${ }^{4}$

$$
D(\omega)=\frac{l^{2}}{6} \frac{\mathrm{i} \omega \tilde{\psi}(\mathrm{i} \omega)}{1-\tilde{\psi}(\mathrm{i} \omega)},
$$

where $l$ is the (frequency independent) root-mean-square jump length. $D$ can be seen to be independent of frequency if $\psi(t)$ is a simple exponential. A combination of eqns. (3) and (4) gives the dispersive resistivity as

$$
\frac{1}{Z_{\mathrm{CTRW}}}=\frac{e}{k T} g n D(\omega) .
$$

If $(1 / \vartheta)$ is given as the zero frequency limit of the frequencydependent term in eqn. (4),

$$
\frac{1}{\vartheta}=\lim _{\omega \rightarrow 0} \frac{\mathrm{i} \omega \tilde{\psi}(\mathrm{i} \omega)}{1-\tilde{\psi}(\mathrm{i} \omega)},
$$

one can recover the classical expression from eqns. (4)-(6) as

$$
\frac{1}{R(\omega \rightarrow 0)}=\frac{e}{k T} g n \frac{l^{2}}{6 \vartheta}
$$

The task is now to compare the experimental data, given in terms of eqn. (2), with the above theoretical expression. Let $Z_{\text {CPER }}$ and $Z_{\text {CTRW }}$ be equated and $\tilde{\psi}(\mathrm{i} \omega)$ expressed through the quantities obtained from the experiments. Then one finds

$$
\tilde{\psi}(\mathrm{i} \omega)=\left[1+\frac{\mathrm{i} \omega \vartheta}{1+(\mathrm{i} \omega \tau)^{\alpha}}\right]^{-1} .
$$

Whereas the inverse transform of eqn. (8) seems difficult to obtain, the low frequency limit for the inverse Fourier transform of $\tilde{\psi}(\mathrm{i} \omega)$ can be evaluated. If the relationship

$$
1 \gg\left|(i \omega \tau)^{\alpha}\right|
$$

holds, a non-dispersive charge carrier mobility may be attained also at finite frequencies. By considering the above inequality eqn.(8) takes the approximate form

$$
\tilde{\psi}_{l f}(\mathrm{i} \omega)=[1+\mathrm{i} \omega \vartheta]^{-1},
$$

which corresponds to an exponential waiting time distribution function for low frequencies,

$$
\psi_{l f}(t)=(1 / \vartheta) e^{-t / \vartheta} .
$$

By combining eqn. (10) with eqns. (4) and (5) one obtains eqn. (7). The average waiting time in this case is $\langle t\rangle_{\mathrm{If}}=\vartheta$.

Also the high frequency limit can be evaluated. If the inequalities

$$
1 \ll|\mathrm{i} \omega \vartheta|, \quad 1 \ll\left|(\mathrm{i} \omega \tau)^{\alpha}\right|
$$

prevail, eqn.(8) can be approximated as

$$
\tilde{\psi}_{\mathrm{hf}}(\mathrm{i} \omega)=\frac{\tau}{\vartheta} \frac{1}{(\mathrm{i} \omega \tau)^{1-\alpha}} \approx \frac{\tau}{\vartheta} \frac{1}{(1+\mathrm{i} \omega \tau)^{1-\alpha}},
$$

where the second approximation can be substantiated numerically. The inverse Fourier transform of the second expression is proportional to the normalised waiting time distribution function, $\psi_{\mathrm{SL}}(t)$, suggested by Scher and $\operatorname{Lax}^{4}$ as

$$
\frac{\vartheta}{\tau} \psi_{\mathrm{hf}}(t)=\psi_{\mathrm{SL}}(t)=\frac{1}{\Gamma(1-\alpha)} \frac{1}{\tau}\left(\frac{t}{\tau}\right)^{-\alpha} \mathrm{e}^{-t / \tau},
$$

where $\Gamma$ denotes the gamma function. The average waiting time for the high frequency transport was evaluated ${ }^{4}$ by eqn.(14) as $\langle t\rangle_{\mathrm{hf}}=(1-\alpha) \tau$.

The high frequency time scale has been found by parameter fitting, $\tau$ being of the order of $10^{-2} \mathrm{~s}$. The low frequency time scale is defined, in terms of eqns. (3) and (7), as the average waiting time characteristic to dc charge carrier motion $\langle t\rangle_{\omega \rightarrow 0}$ which, on the other hand, defines $R_{\mathrm{ox}}$, the dc impedance. The dc limit of any electrode impedance corresponds to a Faraday process i.e. to some chemical transformation which, in the present case, is nothing else but oxide growth. This is controlled by atomic diffusion across the oxide layer already present. If diffusion proceeds in a solid-like manner, because the oxide has a high Deborah number, ${ }^{6}$ the average waiting time is of the order of $1 \mathrm{~s}$ near room temperature. ${ }^{7}$ Hence it seems to be safe to take $\vartheta \cong 1 \mathrm{~s}$. An order of magnitude estimate of $\vartheta$ based on eqn.(7) gives a similar result. Thus the inequality $\tau \ll \vartheta$ holds and the approximations based on eqns. (9) and (12) are valid.

According to the above idea and to elementary notions of electrochemistry, $1 / R_{\mathrm{ox}}$ is regarded as a measure of oxide growth rate. The increase of $1 / R_{\mathrm{ox}}$ with increasing temperature can be attributed to both the increase in mobile charge density, $n$, and the decrease in atomic hopping time, $\vartheta$.

The parameters of CPE, $\tau$ and $\alpha$, are thought to characterise the non-Faraday charge transport across the oxide. The orders of magnitude difference between $\vartheta$ and $\tau$ together with the negligible temperature dependences of both $\alpha$ and $\tau$ show that the charge carriers, which are due to ac conductivity, are different from the atomic carriers which account for $R_{\mathrm{ox}}$. A small decrease in $\alpha$ and the approximate constancy of $\tau$ are in keeping with the trapping model suggested by Scher and Lax: ${ }^{4}$ if the temperature increases the role of charge trapping decreases, hence the charge transport becomes less dispersive.

It has not been our present aim to propose a detailed theory of the temperature dependences of the parameters i.e. to give a quantitative explanation of the findings summarised in Fig.3. We could, however, show that the temperature dependence of the CPE parameter, $\sigma$, is a clear indicator of the underlying mechanism. If it decreases with temperature, CPE can be attributed to some dispersion in dielectric relaxation, whereas in cases like the present one, when it increases with temperature, the constant phase element can be treated in terms of the theory of continuous time random walk.

\section{Conclusions}

The impedance analysis of hydrothermally grown oxide layers on $\mathrm{Zr}-1 \% \mathrm{Nb}$ surfaces showed that the portion of the impedance spectrum attributed to the oxide can be characterised by a parallel CPE $\| R_{\mathrm{ox}}$ equivalent circuit. By studying the temperature dependence of the parameters of the constant phase element (CPE) we concluded that the frequency dependence is due to dispersion in charge carrier mobility that can be described in terms of the continuous time random walk theory. In this way we made a distinction between the kinetics of the Faraday process, i.e. oxide growth, and of the non-Faraday charge transport across the oxide. The average waiting times between successive jumps of the charge carriers differ markedly for the two processes: of the order of $10 \mathrm{~ms}$ for the non-Faraday process compared to about $1 \mathrm{~s}$ for the oxide growth. 


\section{Acknowledgement}

This work was supported by the National Science Research Fund, Hungary, (OTKA) under Contract No. T 29894.

\section{References}

1 A. Lasia, in Modern Aspects of Electrochemistry, ed. B. E. Conway, J. O'M. Bockris and R. A. White, Kluwer, Academic/Plenum, New York, 1999, vol. 32, p.143.
2 (a) G. Nagy, Zs. Kerner, G. Battistig, A. Pintér-Csordás, J. Balog and T. Pajkossy, J. Nucl. Mater., 2001, 297, 62; (b) G. Nagy, Zs. Kerner and T. Pajkossy, J. Nucl. Mater., in press.

3 Impedance Spectroscopy, Emphasizing Solid Materials and Systems, ed. J. Ross Macdonald, Wiley, New York, USA, 1987.

4 (a) H. Scher and M. Lax, Phys. Rev. B, 1973, 7, 4491; (b) H. Scher and M. Lax, Phys. Rev. B, 1973, 7, 4502.

5 (a) A. Compte, Phys. Rev. E, 1996, 53, 4191; (b) J. Bisquert and A. Compte, J. Electroanal. Chem., 2001, 499, 112.

6 S. R. Elliott, The Physics and Chemistry of Solids, Wiley, New York, 1998, p. 170.

7 Ch. Kittel, Introduction to Solid State Physics, Wiley, New York, 6th edn., 1986, p. 520. 